\title{
One Wire Is always Better
}

\author{
Michael Bank, Vladimir Tsingauz \\ Jerusalem College of Technology, Jerusalem, Israel \\ Email: bankmichael1@gmail.com
}

How to cite this paper: Bank, $M$. and Tsingauz, V. (2021) One Wire Is always Better. Engineering, 13, 300-305. https://doi.org/10.4236/eng.2021.136022

Received: May 16, 2021

Accepted: June 22, 2021

Published: June 25, 2021

Copyright $\odot 2021$ by author(s) and Scientific Research Publishing Inc. This work is licensed under the Creative Commons Attribution International License (CC BY 4.0).

http://creativecommons.org/licenses/by/4.0/

\begin{abstract}
The efficiency of transmitting electrical energy from tree phase source is very complicated and expensive. In the same time, it is known that active electrical energy is transmitted from a source to a load and does not come back to the source. If this is so, then why in all electrical devices and systems for transmission of electricity, one uses more than one wire? "It is shown that any electrical system can be made using a one-wire method". The main results were received by several inventions that allow to change three wires line to one wire. The important contribution of the work is refinement calculation of the total power of a three-phase signal. It is showed that in proposed one wire system, the wire transmits a power more than in each wire in three-phase system. The cost of the electrical energy transmitting system can be reduced, also can be improved and the use of the renewable electrical energy can be sped up.
\end{abstract}

\section{Keywords}

One Wire

\section{One Wire Instead of Two}

One can consider that in both wires of a usual two-wire electrical system, there are two currents which have their differences in a polarity only. If this is so, then we can invert one current to an opposite polarity and after that to sum both currents. As a result, we will need now one wire only. Then before a load, we can divide this current into two currents and invert one of them. This approach has been proposed, tested and confirmed by us with many simulations and experiments [1] [2] [3].

An easy method for decreasing the number of wires has been used here by us. If we would like to use instead of two wires only one wire, then we have to make the same phases in each of the wires. After that, the wires can combine with each 
other. This is the structure of the converter 2 - 1 above.

For building of an opposite converter 1 - 2, one can divide one wire into two wires. After that one has to set the required phase values in each wire.

As usual, we are hearing an opinion that since we are using one wire, it is more expensive because the electric current increases twice. The response to this is that we are using only one wire instead of two wires, and as a result, the length of the wires is by two times shorter.

\section{One Wire Instead of Three Using Converter 3 - 1}

The current most popular method for transmitting electrical energy in large quantity is three-phase system. Here we can implement another method for using one wire.

A three-phase signal is a signal which consists of three vectors with phase differences of 120 degrees. The sum of two vectors with phase differences of 120 degrees is one vector with the same amplitude. If we invert the third vector and add it to the vector obtained above, then we obtain a sum of all three vectors (Figure 1 ).

A possible diagram of a converter 3 - 1 which converts a three-phase current into a one-wire current and simulations results are shown in Figure 2.

The sum of three vectors which we are received using three transformers is shown at the left side of Figure 2 [4]. The lower transformer is an inverter its windings are turned on in the opposite direction. The transformer at the right side allows changing a signal level of an output. Therefore, this converter can also work as a step-up transformer at the input of the electric current line to reduce losses in it. Figure 2 also shows simulation results for a frequency $50 \mathrm{~Hz}$.

For zeroing an electrical potential in corresponding places a nullifier [5] is utilized.

The idea of proposed converter 3 - 1 allows to receive new conclusions about summing power of three-phase signal. Usual one can read that transmitting three-phase signal power equals sum powers in signals of all three phases. But this power value one can get on three separated loads.

The concept of the power of a three-phase signal is uncertain, since the total voltage of a three-phase signal, as can be seen from Figure 3, is equal to zero. On the same figure showed that the voltage at the output of the converter $3-1$ is

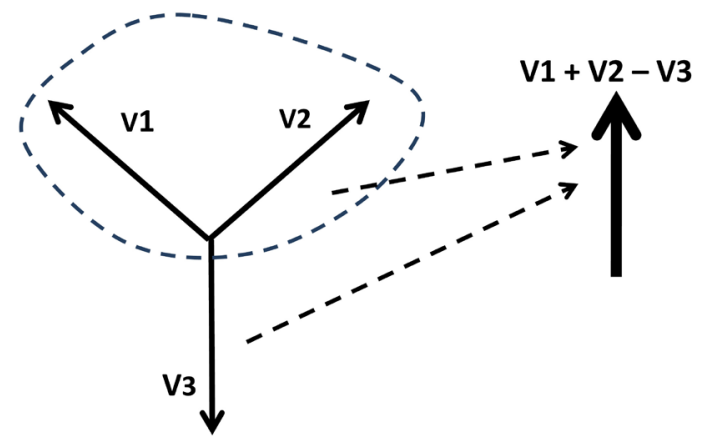

Figure 1. Combining of three vectors. 


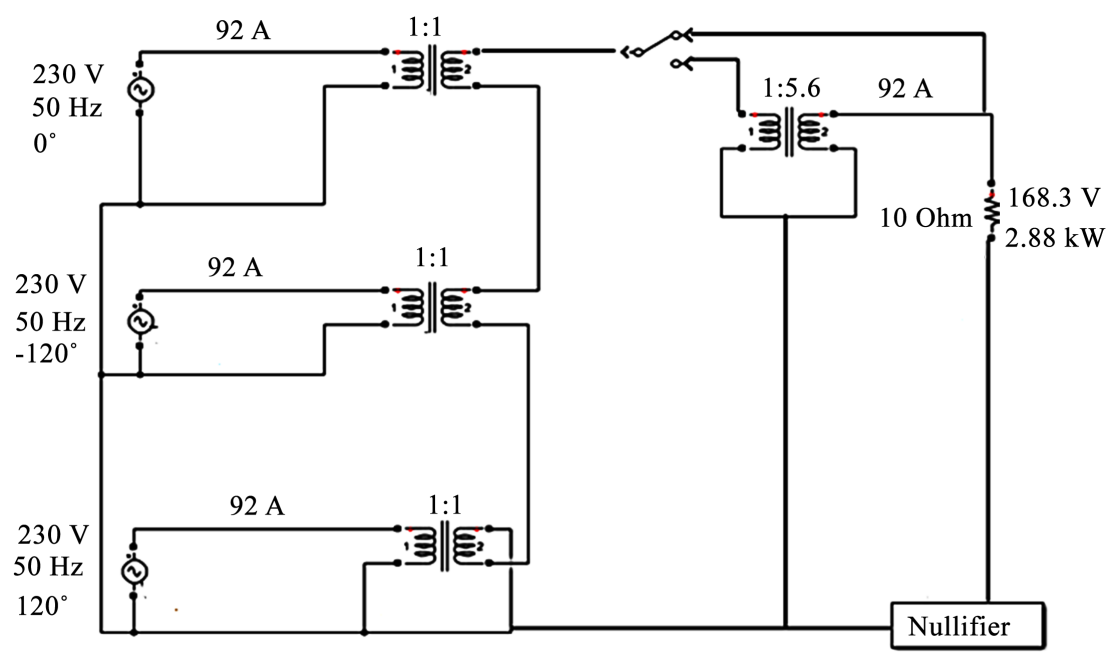

Figure 2. The scheme of converter 3 - 1 and simulations results.

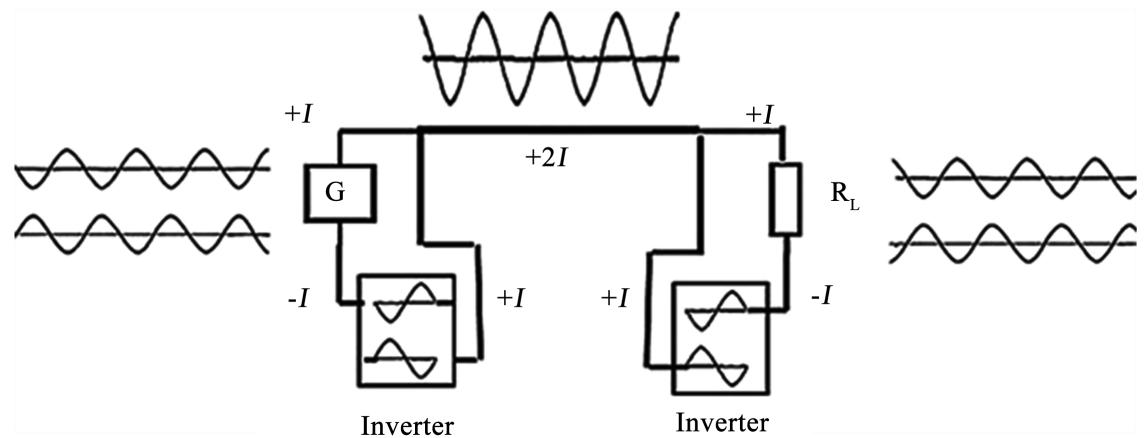

Figure 3. Convertors $2-1$ and $1-2$.

equal to $2 \mathrm{~V}$, where $\mathrm{V}$ is one phase voltage. In other words one wire method allows getting three phase signal power to one load. And this power will be equals sum of two powers one-phase signal.

Below on Figure 5 will show example using three-phase signal power in system for accumulator charging.

\section{Converter 1 - 3}

There are many devices which use a three-phase signal. If one must give to this device one wire signal, he must use a converter $1-3$. The example of this case is an electrical car charging station, which is working with a three-phase signal. For this station a special converter $1-3$ shown in Figure 4 was built.

The converter 1 - 3 which converts a one-wire current into a three-phase current and simulations results are shown in Figure 4. Here one electric signal is divided into three signals. Two lower signals receive phase shifts by sequence resonance circuits with resonance frequencies higher and lower than $50 \mathrm{~Hz}$. The lower transformer is an inverter.

This converter must take into consideration that an accumulator can change its input resistance during charging process. This particularity was taken into 
account when developing this converter. In Table 1 it can be seen that a converter output power is about constant with an input resistance changing from 40 to 60 Ohm.

\section{Converter 1 - DC}

One of the problems with fast charging on highways is a need of high voltage and a great power. However, this is not always available on a road.

Nowadays in many cases a three-phase electric signal is rectified by complicated devices and after that it is served in a car accumulator.

In [6] the following method for roadway conditions is disclosed. Only one electric wire is placed under asphalt. This allows building charging stations over any significant distances.

The fastest charging can be provided by using DC as an input signal. For this purpose a converter 1 - DC which is shown in Figure 5 can be used.

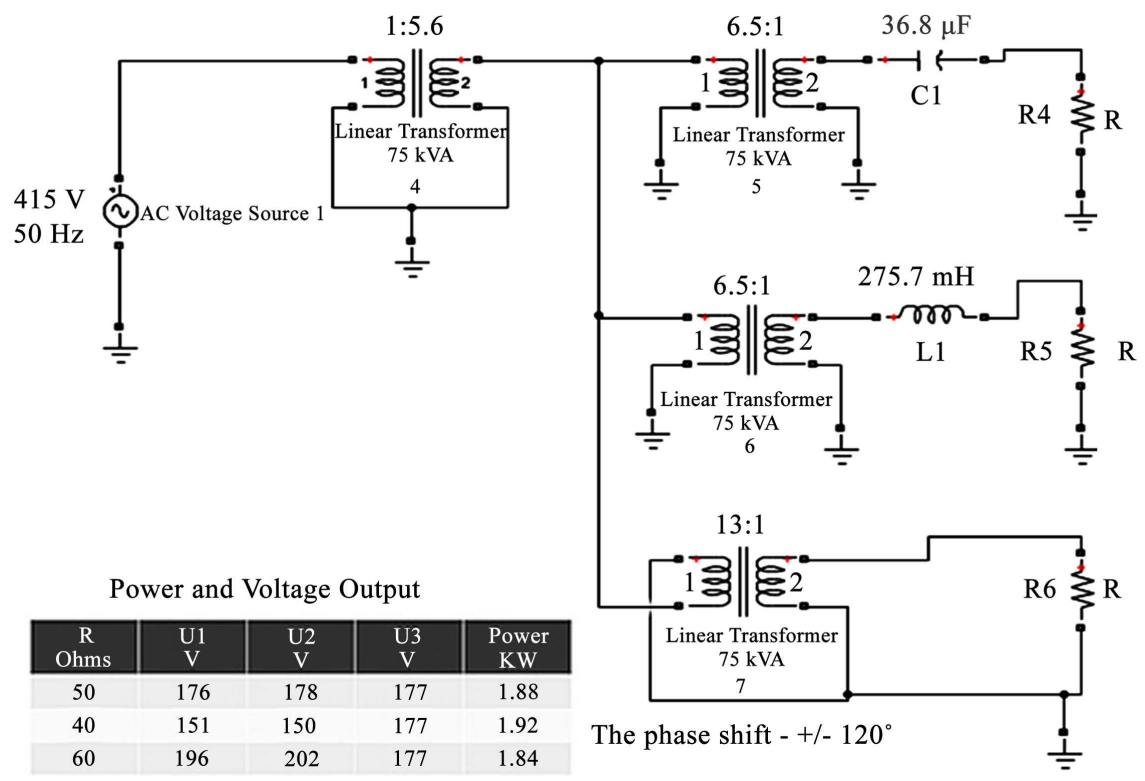

Figure 4. The scheme of converter 1 - 3 and simulations results.

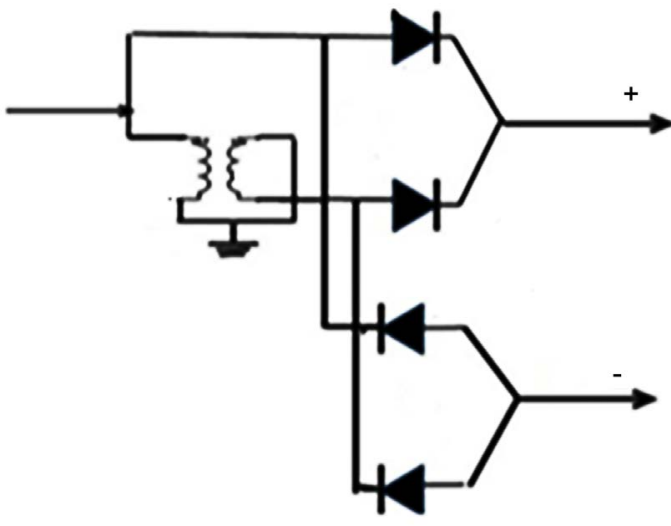

Figure 5. The scheme of converter 1 - DC. 
Table 1. The output power in converter 1 - 3 with different loads as simulations results.

\begin{tabular}{ccccc}
\hline \multirow{2}{*}{ R.Ohms } & \multicolumn{3}{c}{ Voltage Output } & \multirow{2}{*}{ Power KW } \\
\cline { 2 - 4 } & $\mathrm{U} 1 \mathrm{~V}$ & $\mathrm{U} 2 \mathrm{~V}$ & $\mathrm{U} 3 \mathrm{~V}$ & 1.88 \\
\hline 50 & 178 & 176 & 177 & 1.89 \\
40 & 149.7 & 147.5 & 177.1 & 1.88 \\
\hline 60 & 203 & 200.6 & 177.1 & \\
\hline
\end{tabular}

\section{Conclusions}

Russian scientist Dolivo-Dobrovolsky in 1890 developed and demonstrated a three-phase current system. He was awarded the Gold Medal of the World Exhibition in Paris (1900) for his achievements in the field of electrical engineering. Today, this is what is many times written on the Internet in the sections describing the three-phase system:

In https://www.pergam.ru/articles/trehfazny-tok.htm, one can read: "What are the advantages of a three-phase generation and power supply system? Savings on the number of wires needed to transfer electricity. Given the considerable distances (hundreds and thousands of kilometers) and the fact that non-ferrous metals with low electrical resistivity are used for wires, the savings are very significant."

Also in

http://www.electricalbasicprojects.com/three-phase-system-advantages-over-sin gle-phase-system it is stated: "Transmitting the same amount of power at the same voltage, a three-phase transmission line requires less conductor material than a single-phase line. The three-phase transmission system is so cheaper". A lot of similar quotes can be found.

Nowadays, about 130 years later, in most cases, electrical energy is transmitted by a three-phase method. In [1] [2] and other articles and patents, it has been shown by us that all the energy which is transmitted in a three-phase system can be transmitted instead through one wire and without the participation of grounding. This single wire can be one of the wires of the compared three-phase system. In addition, the single-wire method does not require expensive intermediate stations for reactive power compensation and bulky supports. This method allows to transfer electrical energy underground or underwater. It has been proven that a single-wire system is several times cheaper than a three-phase system.

At the end of the article, we note once again. If you use one wire instead of several wires in a device or in a system for transmitting electrical energy or information, you will get the same results and this despite the fact that the only wire is utilized which will be exactly the same as the two or three wires that you gave up, [1], page 57.

The converter 3 - 1 in one-wire method allows getting total three-phase power on one load.

In a final conclusion of this article, the authors would like to propose to disas- 
semble all multi-wire electrical systems and replace them with new single-wire systems which have enormous advantages.

Of course, it is not possible to do this quickly.

Well, we have to wait, and eventually, no doubts, this will happen.

\section{Acknowledgements}

The authors are grateful to Yuri Shalyt, who made a number of simulations.

\section{Conflicts of Interest}

The authors declare no conflicts of interest regarding the publication of this paper.

\section{References}

[1] Bank, M. (2012) Single-Wire Electric Transmission Line. US Patent No. 9,608,441.

[2] Bank, M. (2017) It Is Quite Another Electricity: Transmitting by One Wire and without Grounding. Patridge.

[3] Bank, M. (2012) New One-Way Line for Electric Transmission System. Journal of Energy and Power Engineering, 6, 1320-1327.

[4] Bank, M. (2017) Phase Converter for Vector Conversion of Three Phase Signals. US Patent No. 10,305,289.

[5] Bank, M. (2020) Zeroing Instead of Grounding. Journal of Energy and Power Engineering, 14, 33-37. https://doi.org/10.17265/1934-8975/2020.01.004

[6] Bank, M. (2018) System for Charging Electrically Drive Vehicles with a Single Line for Transmitting Electric Current from a Source to a Charging Station. U.S. Patent No. 10,250,061. 